\title{
Delayed meconium passage associated with early-onset neonatal cystitis
}

\author{
Taheri $\mathbf{P A}^{* 1}$ \\ ${ }^{1}$ Neonatologist, Neonatal Department, Tehran University of Medical Sciences, Tehran, Iran
}

\begin{abstract}
Failure to pass meconium after 48 hours of life in a full-term newborn may be a sign of serious conditions and obstructive gastrointestinal disorders should be ruled out. We report a rare case of delayed meconium passage in a term newborn with moderate abdominal distention and lower urinary tract infection. After 18 hours of antibiotic treatment, abdominal distention was reduced and meconium passage occurred. This case showed that early-onset cystitis may lead to delayed meconium passage.
\end{abstract}

\section{Introduction}

Most premature and almost all term neonates pass the first meconium during the first 48 hours of life [1,2]. Failure to pass meconium within the first 48 hours of life in a full-term newborn may be a sign of gastro-intestinal obstructive conditions (such as Hirschprung's disease, gastro-intestinal malformations, ...) or non-obstructive disorders (such as neonatal sepsis, asphyxia, hypothyroidism, ...) [3-6]. Failure to pass meconium along with abdominal distension and bilious vomiting are the classic clinical manifestations of intestinal obstruction in neonates. Anal examination is necessary to rule out ano-rectal stenosis, peri-anal fistula and anal atresia [5].

We report a rare case of delayed meconium passage in a term newborn that was found to have lower urinary tract infection and discuss the possible underlying mechanism. To our knowledge, this is the first case of delayed meconium passage associated with an infection localized to lower urinary tract in a term newborn.

\section{Case report}

A two-day old, term, male infant was admitted to the neonatal ward of Bahrami Children Hospital with a history of delayed meconium passage since birth and mild abdominal distention. The infant was delivered via cesarean section at 38 weeks of gestational age with the 1st minute Apgar score of 9/10.There was no history of maternal diseases, drug addiction or medication taking either before or during pregnancy. He had no physical anomaly and there was no family history of congenital defects. No history of bilious or non-bilious vomiting was mentioned by the parents.

On Physical examination, the neonate was alert and afebrile with normal vital signs. Except for mild distention of the abdomen, no other significant finding was detected. Ano-rectal examination was normal. Prone abdominal X-ray showed dilated intestinal loops with normal gas distribution to anorectal area. The results of laboratory tests were as follows: Total Leucocyte Count (TLC) $11.9 \times 1000 / \mathrm{mm}^{3}$ with $64 \%$ Neutrophils, 33\% Lymphocytes, 2\% Monocytes and 1\% Eosinophils, Hemoglobin (Hb) $16.9 \mathrm{gr} / \mathrm{dl}$, platelet count $205 \times 1000 / \mathrm{mm}^{3}$, C-Reactive Protein (CRP) $3 \mathrm{mg} / \mathrm{l}$, Erythrocyte Sedimentation Rate (ESR) $1 \mathrm{~mm} / \mathrm{hr}$. Biochemical indices were normal.
In view of infant's abdominal distention and persistence of delayed meconium passage, sepsis work up was performed and intravenous antibiotic, including ampicillin and gentamicin, was started. The sample of suprapubic urine culture revealed 30.000 Escherichia coli (E. coli) colonies sensitive to cefixime, ceftriaxone, amikacin and gentamicin. After 18 hours of treatment, meconium passage occurred and abdominal distention subsided. Oral feeding was started as soon as the patient became clinically stable.

The second urine culture was negative 48 hours after antibiotic administration. Voiding cystourethrogram (VCUG) was performed and revealed normal results. He was discharged after seven days of hospitalization. Regular follow-up visits till two months of age showed normal growth indices, feeding and bowel habits.

\section{Discussion}

Timely meconium passage is a sign of newborns' well-being [5]. Ninety five percent of healthy, term infants pass their first meconium within 48 hours [7]. Delayed meconium passage (generally defined as non-passage within the first 48 hours after birth) is mainly a condition of preterm infants and is reported in up to one third of preterm and low birth weight infants due to factors such as delayed maturation of colorectal motility mechanisms, composition of meconium, severe Respiratory Distress Syndrome (RDS), and delayed enteral feeding [7].

In case of failure to pass meconium in term infants, obstructive disorders (such as meconium ileus, colonic atresia, malrotation and enteric nervous system anomalies), maternal drug taking (such as illicit drugs, magnesium sulfate and ganglionic blocking agents) and systemic conditions of the newborn (e.g. hypothyroidism, hypercalcemia,

Correspondence to: Peymaneh Alizadeh Taheri, Bahrami Children Hospital, Tehran, Iran; Tel: +98 21 73013420; Fax: +98 21 77568809; E-mail: p.alizadet@ yahoo.com

Key words: delayed meconium passage, term newborn, early-onset cystitis

Received: February 25, 2017; Accepted: March 19, 2017; Published: March 23 2017 
hypokalemia, sepsis and congestive heart failure) should be evaluated [1]. No relationship has been found between feeding type (breast milk or formula) and delayed passage of meconium [8].

The infant reported by authors was the result of an uneventful pregnancy. There has been no history of maternal disease or medication taking. The newborn was term and born via cesarean-section with a normal Apgar score. There was no history of vomiting. Physical examination and abdominal X-ray showed no signs of gastrointestinal obstructive disorders. Although proved to have urinary tract infection, the infant was afebrile and medically stable, with no signs or laboratory findings indicative of sepsis. Abdominal distension and cystitis responded to antibiotic administration, followed by meconium passage. This process suggests that failure to meconium passage had probably been due to relative ileus secondary to cystitis.

This case shows that urinary tract infection without sepsis may induce delayed meconium passage in neonatal period.

\section{Acknowledgement}

The author wishes to thank the nursing staff of neonatology ward and the Research Development Center of Bahrami Children Hospital.

\section{References}

1. Clark DA (1977) Times of first void and first stool in 500 newborns. Pediatrics 60 457-459. [Crossref]

2. Martin RJ, Fanaroff AA, Walsh MC (2015) Fanaroff and Martin's Neonatal-Perinata Medicine diseases of the fetus and infant.10th ed, Elsevier, Saunders, Philadelphia, USA.

3. Bekkali N, Hamers SL, Schipperus MR, Reitsma JB, Valerio PG, et al. (2008) Duration of meconium passage in preterm and term infants. Arch Dis Child Fetal Neonatal Ed 93: F376-379. [Crossref]

4. Ringer SA, Hansen AR (2012) Surgical emergencies in the newborn. In: Cloherty JP, Eichenwald EC, Hamsen AR, Stark AR. (eds.) Manual of neonatal care.7th ed, Wolters Kluwer, Lippincott Williams and Wilkins, Philadelphia, USA. p. 808-830.

5. Loening-Baucke V, Kimura K (1999) Failure to pass meconium: diagnosing neonatal intestinal obstruction. Am Fam Physician 60: 2043-2050. [Crossref]

6. Sanchéz LL, Lugo-Vicente H (2010) Failure to pass meconium. Bol Asoc Med P R 102: 62-67. [Crossref]

7. Gulcan H, Gungor S, Tiker F, Kilicdag H (2006) Effect of perinatal factors on time of first stool passage in preterm newborns: An open, prospective study. Curr Ther Res Clin Exp 67: 214-225. [Crossref]

8. Metaj M, Laroia N, Lawrence RA, Ryan RM (2003) Comparison of breast- and formula-fed normal newborns in time to first stool and urine. $J$ Perinatol 23: 624-628. [Crossref]

Copyright: (C2017 Taheri PA. This is an open-access article distributed under the terms of the Creative Commons Attribution License, which permits unrestricted use, distribution, and reproduction in any medium, provided the original author and source are credited. 